\title{
Rough maximal functions and rough singular integral operators applied to integrable radial functions
}

\section{Peter Sjögren and Fernando Soria}

\begin{abstract}
Let $\Omega$ be homogeneous of degree 0 in $\mathbb{R}^{n}$ and integrable on the unit sphere. A rough maximal operator is obtained by inserting a factor $\Omega$ in the definition of the ordinary maximal function. Rough singular integral operators are given by principal value kernels $\Omega(y) /|y|^{n}$, provided that the mean value of $\Omega$ vanishes. In an earlier paper, the authors showed that a two-dimensional rough maximal operator is of weak type $(1,1)$ when restricted to radial functions. This result is now extended to arbitrary finite dimension, and to rough singular integrals.
\end{abstract}

\section{Introduction.}

Let $\Omega \geq 0$ be an integrable function on the unit sphere $S^{n-1}$ in $\mathbb{R}^{n}$, and extend it to a function in $\mathbb{R}^{n} \backslash\{0\}$, homogeneous of degree 0 . The rough maximal operator corresponding to $\Omega$ is defined by

$$
M_{\Omega} f(x)=\sup _{r>0} \frac{1}{r^{n}} \int_{|y|<r} \Omega(y)|f(x-y)| d y, \quad f \in L_{\mathrm{loc}}^{1}\left(\mathbb{R}^{n}\right) .
$$

This operator is bounded on $L^{p}\left(\mathbb{R}^{n}\right), 1<p<\infty$, as seen by the method 
of rotations. It is, however, unknown whether it is of weak type $(1,1)$.

Under (weak) additional assumptions on $\Omega$, several authors have proved the weak type; see the authors' paper [S-S] for details. That paper contains a proof that $M_{\Omega}$ is of weak type $(1,1)$ in the plane when restricted to radial functions $f$, for a general $\Omega \in L^{1}$. In fact, the same result is proved for $n=2$ when $M_{\Omega}$ is replaced by the larger operator

$$
M_{\Omega}^{*} f(x)=\int_{S^{n-1}} \Omega(\omega) M_{\omega} f(x) d \omega
$$

Here and below, $d \omega$ is the area measure on $S^{n-1}$. Further, $M_{\omega}$ is the one-dimensional maximal operator in the direction $\omega \in S^{n-1}$, defined by

$$
M_{\omega} f(x)=\sup _{r>0} \frac{1}{r} \int_{0}^{r}|f(x-t \omega)| d t .
$$

As we pointed out in $[\mathrm{S}-\mathrm{S}], M_{\Omega}^{*}$ cannot be of weak type $(1,1)$ on general functions even when $\Omega$ is the constant function 1 . In this paper, we shall extend the above to $\mathbb{R}^{n}$, as follows.

Theorem 1. The operator $M_{\Omega}^{*}$ is of weak type $(1,1)$ when restricted to radial functions in $\mathbb{R}^{n}$, for any nonnegative $\Omega \in L^{1}\left(S^{n-1}\right)$ and any $n$. The same is true for $M_{\Omega}$.

Rough singular integral operators can be defined analogously. Now $\Omega \in L^{1}\left(S^{n-1}\right)$ must have mean value 0 . Let

$$
T_{\Omega} f(x)=\text { p.v. } \int \frac{\Omega(y)}{|y|^{n}} f(x-y) d y=\lim _{\substack{\varepsilon \rightarrow 0 \\ R \rightarrow \infty}} \int_{\varepsilon<|y|<R} \frac{\Omega(y)}{|y|^{n}} f(x-y) d y,
$$

whenever the limit exists. The $L^{p}$ boundedness of such operators (which is easy when $\Omega$ is odd) was proved by Calderón and Zygmund [C-Z] assuming $\Omega \in L \log L\left(S^{n-1}\right)$. There is a nice proof due to J. Duoandikoetxea and J. L. Rubio de Francia [D-RF] when $\Omega \in L^{q}\left(S^{n-1}\right)$, $q>1$. With the same condition on $\Omega$, S. Hofmann $[\mathrm{H}]$ proved the weak type $(1,1)$ in the plane. The same was proved for $\Omega \in L \log L\left(S^{n-1}\right)$ by M. Christ and J. L. Rubio de Francia [Ch-RF]. In an unpublished work, they also extended the result to dimension at most 7. More recently, A. Seeger [Se] has proved it in any dimension, again under the hypothesis $\Omega \in L \log L\left(S^{n-1}\right)$. We remark that the $L^{p}$ inequality, $1<p<\infty$, cannot hold without additional assumptions on $\Omega$, since 
the Fourier multiplier corresponding to $T_{\Omega}$ need not be bounded ( $c f$. [St, Chapter II]). In our result, we have no additional assumption on $\Omega$, but apply the operator only to radial functions.

Theorem 2. Let $\Omega \in L^{1}\left(S^{n-1}\right)$ with $\int_{S^{n-1}} \Omega d \omega=0$. The operator $T_{\Omega} f$ is well defined for any radial function $f \in L^{p}\left(\mathbb{R}^{n}\right), 1 \leq p<\infty$, in the sense that the principal value exists for almost every $x$. Moreover, when restricted to radial functions, $T_{\Omega}$ is of weak type $(1,1)$ and bounded on $L^{p}, 1<p<\infty$, and so is the maximal singular integral operator

$$
T_{\Omega}^{*} f(x)=\sup _{0<\varepsilon<R<\infty}\left|\int_{\varepsilon<|y|<R} \frac{\Omega(y)}{|y|^{n}} f(x-y) d y\right| .
$$

To prove the two-dimensional estimate for $M_{\Omega}^{*}$ in [S-S, Theorem 3], we applied Theorems 1 and 2 of $[\mathrm{S}-\mathrm{S}]$. These two results say that $y^{-1} G(y \cdot) * f(x) \in L^{1, \infty}(y d x d y, y>0)$ for any $f \in L^{1}(\mathbb{R})$ and suitable $G \in L^{1}(\mathbb{R})$. Our method to prove Theorem 1 in the present paper is similar. Implicit in our proof is a version of Theorem 2 of [S-S], where $\mathbb{R}$ is replaced by $S^{n-1}$. We point out that a version with $\mathbb{R}$ replaced by $\mathbb{R}^{n}$ also follows from the arguments below. However, we leave it to the interested reader to state it explicitly.

Theorem 1 of this paper is proved in Section 2. It is then one of the tools used to prove Theorem 2 in Section 3.

Finally, with respect to the notation in this paper, an integral $\int_{a}^{b}$ with $a>b$ should be interpreted as 0 . Further, $C$ denotes many different positive finite constants.

\section{Proof of Theorem 1.}

We write $x \in \mathbb{R}^{n}$ as $x=r \theta$ with $r \geq 0$ and $\theta \in S^{n-1}$, and denote as in [S-S] by $A(\omega, \theta)=A(\omega, x) \in[0, \pi)$ the angle between $\omega \in S^{n-1}$ and $\theta$. Also let $s(\omega, \theta)=\max \{\sin A(\omega, \theta), A(\omega, \theta) / 2\}$. With $0 \leq g \in L^{1}\left(t^{n-1} d t\right)$ defined on $\mathbb{R}_{+}$, we follow [S-S] in defining

$$
A_{\omega} g(x)=\frac{1}{r} \int_{r s(\omega, \theta)}^{\infty} g(t) \frac{t d t}{\left(t^{2}-r^{2} s(\omega, \theta)^{2}\right)^{1 / 2}} .
$$

Consider the operator

$$
P g(x)=\int_{s(\omega, \theta)<\delta} \Omega(\omega) A_{\omega} g(x) d \omega,
$$


where $\delta>0$ will be a small constant. The first part of the proof of [S-S, Theorem 3], which is carried out for each $n \geq 2$, now shows that we need only find an estimate

$$
P: L^{1}\left([0, \infty), t^{n-1} d t\right) \longrightarrow L^{1, \infty}\left(S^{n-1} \times[0, \infty), r^{n-1} d \theta d r\right)
$$

Notice that

$$
A_{\omega} g(x) \leq \frac{1}{r} G(r s(\omega, \theta))
$$

where

$$
G(u)=\int_{u}^{\infty} g(t) \frac{t^{1 / 2} d t}{(t-u)^{1 / 2}}, \quad u>0 .
$$

Essentially as in [S-S, proof of Theorem 3], we majorize $G$ by

$$
G \leq C \sum_{\nu=0}^{\infty} 2^{-\nu / 2} G_{\nu}+C h
$$

where

$$
\begin{aligned}
& G_{\nu}(u)= 2^{\nu} u^{1-n} \int_{u}^{2^{2^{-\nu}} u} g(t) t^{n-1} d t \\
& \leq C \sum_{k \in \mathbb{Z}} \int_{2^{-k 2^{-\nu}}}^{2^{(2-k) 2^{-\nu}}} g(t) t^{n-1} d t \\
& \cdot 2^{\nu} 2^{k 2^{-\nu}(n-1)} \chi_{\left[2^{-k 2^{-\nu}}, 2^{(1-k) 2^{-\nu}}\right]}(u)
\end{aligned}
$$

and

$$
h(u)=\int_{u}^{\infty} g(t) d t .
$$

This implies

$$
\begin{aligned}
P g(x) \leq & C \sum_{\nu=0}^{\infty} 2^{-\nu / 2} r^{-1} \int_{s(\omega, \theta)<\delta} \Omega(\omega) G_{\nu}(r s(\omega, \theta)) d \omega \\
& +C r^{-1} \int_{s(\omega, \theta)<\delta} \Omega(\omega) h(r s(\omega, \theta)) d \omega \\
= & C \sum_{\nu=0}^{\infty} 2^{-\nu / 2} P_{\nu} g(x)+C Q g(x),
\end{aligned}
$$


the last equality defining $P_{\nu}$ and $Q$.

To extend the technique used to control $P_{\nu}$ and $Q$ from [S-S], we need analogues of dyadic cubes in $S^{n-1}$. First, we divide $S^{n-1}$ into a finite number of disjoint subsets $E_{s}, s=1, \ldots, s_{0}$, with piecewise smooth boundaries and of small diameters. In each $E_{s}$, we can then introduce coordinates simply by projecting $E_{s}$ orthogonally onto a hyperplane of $\mathbb{R}^{n}$ tangent to $E_{s}$ at some point of $E_{s}$. In this hyperplane, i.e. in $\mathbb{R}^{n-1}$, we introduce the ordinary hierarchy of dyadic cubes. Thus for each $j \in \mathbb{Z}$, we have a partition of $\mathbb{R}^{n-1}$ into cubes of side $2^{-j}$. Some of these cubes have images in $E_{s}$ under the inverse projection. These images will be denoted $\left(I_{j}^{i}\right)_{i}$ and called $2^{-j}$-cubes. This is for $j \geq j_{0}$, some $j_{0}$. Suitably adapted near $\partial E_{s}$, all these sets will form a hierarchy of partitions of $E_{s}$ and, hence, of $S^{n-1}$.

The conditional expectation at level $j, j \geq j_{0}$, of a function $f \in$ $L^{1}\left(S^{n-1}\right)$ is now defined by

$$
E_{j} f(x)=\left|I_{j}^{i}\right|^{-1} \int_{I_{j}^{i}} f, \quad x \in S^{n-1},
$$

where $I_{j}^{i}$ is that $2^{-j}$-cube in $S^{n-1}$ which contains the given point $x$.

Now consider $Q$. The desired estimate

$$
Q: L^{1}\left(t^{n-1} d t\right) \longrightarrow L^{1, \infty}\left(r^{n-1} d \theta d r\right)
$$

can be seen as a version of Theorems 1 and 4 of [S-S], where $\mathbb{R}$ and $\mathbb{R}^{n}$, respectively, are replaced by $S^{n-1}$. Instead of a convolution, we now have the integral defining $Q g$ in (2.2). However, the proof technique carries over without problems. We can assume that the decreasing function $h$ has the form $h=\sum a_{k} \chi_{\left[0,2^{-k-C}\right]}$. Also, it is enough to consider dyadic values of $r$ ( $c f$. the inequality (2.3) below). One can now easily relate $Q$ to the conditional expectation, essentially as in [S-S]. The estimates needed for conditional expectation carry over. This takes care of $Q$.

To control the operator $P$, we must also estimate the $P_{\nu}$. It is enough to prove that each $P_{\nu}$ maps $L^{1}\left(t^{n-1} d t\right)$ boundedly into $L^{1, \infty}\left(r^{n-1} d \theta d r\right)$, with a constant that grows only polynomially in $\nu$. This will allow summing in $L^{1, \infty}$. As in the proof of Theorem 2 in [S-S], we let $r$ take only the values $r=2^{2 \nu j}, j \in \mathbb{Z}$, and prove that

$$
\begin{aligned}
\sum_{j} 2^{2 \nu j n}\left|\left\{\theta \in S^{n-1}: P_{\nu} g\left(2^{2 \nu j} \theta\right)>\lambda\right\}\right| & \\
& \leq C(1+\nu)^{C} \frac{1}{\lambda}\|g\|_{L^{1, \infty}\left(t^{n-1} d t\right)} .
\end{aligned}
$$


Here $|\cdot|$ is the area measure of $S^{n-1}$. This will complete the proof. To verify (2.3), it is enough, as in [S-S, proof of Theorem 2], to sum in (2.1) only over those $k$ of the form $k=\ell 2^{\nu+1} \nu+\kappa, \ell \in \mathbb{Z}$, for each $\kappa=0, \ldots, 2^{\nu+1} \nu-1$. For simplicity, we shall consider only $\kappa=0$. The level set in (2.3) will thus be replaced by the set of those $\theta \in S^{n-1}$ for which

$$
\begin{array}{r}
2^{-2 \nu j} \int_{s(\omega, \theta)<\delta} \Omega(\omega)\left(\sum_{\ell} \int_{2^{-2 \nu \ell}}^{2^{2^{1-\nu}-2 \nu \ell}} g(t) t^{n-1} d t\right) \\
\cdot 2^{\nu} 2^{2(n-1) \nu \ell} \chi_{R_{\ell+j}(\theta)}(\omega) d \omega>\lambda
\end{array}
$$

where $R_{m}(\theta)$ is the ring

$$
R_{m}(\theta)=\left\{\omega \in S^{n-1}: 2^{-2 \nu m} \leq s(\omega, \theta) \leq 2^{2^{-\nu}-2 \nu m}\right\}
$$

Because of the condition $s(\omega, \theta)<\delta$ in the integral in (2.4), we need only consider $m \geq m_{0}$ here, for some $m_{0}>0$. This means that the sum in (2.4) is taken over $\ell \geq m_{0}-j$. Notice that the radius and the width of $R_{m}(\theta)$ are approximately $2^{-2 \nu m}$ and $2^{-\nu-2 \nu m}$, respectively. and form

Next, we let the point $\theta$ move within a $2^{-\nu(1+2 m)}$-cube $I_{\nu(1+2 m)}^{i}$

$$
R_{m}^{i}=\bigcup_{\theta \in I_{\nu(1+2 m)}^{i}} R_{m}(\theta) .
$$

This set is contained in a ring of width at most $C 2^{-\nu(1+2 m)}$. Clearly, $R_{m}^{i}$ is covered by those $2^{-\nu(1+2 m)}$-cubes intersecting it. Their number is at most $C 2^{(n-2) \nu}$. Among these $2^{-\nu(1+2 m)}$-cubes, we discard those which are not in the same $E_{s}$ as $I_{\nu(1+2 m)}^{i}$. Then we enumerate the remaining ones as $I_{\nu(1+2 m)}^{\lambda(i, q)}, q=1, \ldots, q_{0}=\mathrm{O}\left(2^{(n-2) \nu}\right)$, in a coherent way as $i$ varies. By this we mean that the direction from the midpoint of $I_{\nu(1+2 m)}^{i}$ (which is the approximate centre of the ring-like set $R_{m}^{i}$ ) to the midpoint of $I_{\nu(1+2 m)}^{\lambda(i, q)}$ should not vary too much with $i$, for a fixed $q$. It is enough if two such directions never form an angle greater than $\pi / 4$, say, measured in the coordinate system of each $E_{s}$.

In (2.4), we shall now replace $R_{\ell+j}(\theta)$ by $I_{\nu(1+2 \ell+2 j)}^{\lambda(i, q)}$ when $\theta \in$ $I_{\nu(1+2 \ell+2 j)}^{i}$, for a fixed $q$. More precisely, this means that the level set 
in (2.3) is replaced by the union of those $I_{\nu(1+2 \ell+2 j)}^{i}$ for which

$$
\begin{gathered}
2^{-2 \nu j} \int \Omega(\omega)\left(\sum_{\ell \geq m_{0}-j} \int_{2^{-2 \nu \ell}}^{2^{2^{1-\nu}-2 \nu \ell}} g(t) t^{n-1} d t\right) \\
\cdot 2^{\nu} 2^{2(n-1) \nu \ell} \chi_{I_{\nu(1+2 \ell+2 j)}^{\lambda(i, q)}}(\omega) d \omega>\lambda .
\end{gathered}
$$

This version of (2.3), call it (2.3'), implies the theorem, since we can sum in $q$ by means of the adding-up lemma in $L^{1, \infty}$ as in [S-S].

The mean value of $\Omega$ in $I_{\nu(1+2 \ell+2 j)}^{\lambda(i, q)}$ can be seen as an $S^{n-1}$ version of the translated conditional expectation from the proof of Theorem 2 of [S-S]. In fact, the arguments used in that proof now carry over and prove $\left(2.3^{\prime}\right)$. We leave the details to the reader. This ends the proof of Theorem 1.

\section{Proof of Theorem 2.}

We start with the $L^{1}$ case. Let

$$
T_{\Omega}^{\varepsilon, R} f(x)=\int_{\varepsilon<|y|<R} \frac{\Omega(y)}{|y|^{n}} f(x-y) d y .
$$

Notice that all the conclusions follow from the weak type estimate for the maximal operator $T_{\Omega}^{*}$. Also, in the definition of $T_{\Omega}^{*} f(x)$, we need only take $R \geq 10|x|=10 \rho$. This is because in the case $R<10 \rho$, one has

$$
\int_{\varepsilon<|y|<R} \frac{\Omega(y)}{|y|} f(x-y) d y=T_{\Omega}^{\varepsilon, 10 \rho} f(x)-T_{\Omega}^{R, 10 \rho} f(x) .
$$

Together with $T_{\Omega}^{\varepsilon, R}$, we consider

$$
\tilde{T}_{\Omega}^{\varepsilon, R} f(x)=\int_{\substack{|y-x|-|x||>\varepsilon\\| y \mid<R}} \frac{\Omega(y)}{|y|^{n}} f(x-y) d y .
$$

We shall estimate the difference between these two operators.

The notation $x=\rho \theta, y=r \omega, A=A(\theta, \omega)$ will be as in Section 2. A radial function $f \in L^{1}$ will be written $f(x)=g(|x|)$, with $g \in$ $L^{1}\left(\mathbb{R}_{+} ; \rho^{n-1} d \rho\right)$. The distance $t=|x-y|$ satisfies

$$
t^{2}=\rho^{2}+r^{2}-2 \rho r \cos A \text {. }
$$


Hence,

$$
r=\rho \cos A \pm \sqrt{t^{2}-\rho^{2} \sin ^{2} A}
$$

Proposition 3. The operator

$$
\tilde{T}_{\Omega}^{*} f(x)=\sup _{\substack{\varepsilon>0 \\ R>10|x|}}\left|\tilde{T}_{\Omega}^{\varepsilon, R} f(x)\right|,
$$

is of weak type $(1,1)$ when restricted to radial functions.

Proposition 4. The operator

$$
D_{\Omega}^{*} f(x)=\sup _{\substack{\varepsilon>0 \\ R>10|x|}}\left|T_{\Omega}^{\varepsilon, R} f(x)-\tilde{T}_{\Omega}^{\varepsilon, R} f(x)\right|,
$$

is of weak type $(1,1)$ when restricted to radial functions.

It is clear that the $L^{1}$ part of Theorem 2 follows from these two results.

Proof of Proposition 3. In the integral defining $\tilde{T}_{\Omega}^{\varepsilon, R} f(x)$, we pass to polar coordinates, getting

$$
\tilde{T}_{\Omega}^{\varepsilon, R} f(x)=\int_{S^{n-1}} \Omega(\omega) d \omega \int_{\substack{|x-r \omega|-\rho \mid>\varepsilon \\ 0<r<R}} \frac{g(|x-r \omega|)}{r} d r .
$$

Next, we shall transform the inner integral here, using $t=|x-r \omega|$ as a new variable of integration. One has $d r=t d t /(r-\rho \cos A)$. The correspondence between $r$ and $t$ is not quite one-to-one, and the sign in (3.3) must be chosen correctly. As seen geometrically, one obtains a sum of four integrals. Indeed,

$$
\begin{aligned}
& \tilde{T}_{\Omega}^{\varepsilon, R} f(x)=\int_{A>\pi / 2} \Omega(\omega) d \omega \int_{\rho+\varepsilon}^{R_{1}(\rho)} \frac{g(t)}{\rho \cos A+\sqrt{t^{2}-\rho^{2} \sin ^{2} A}} \\
& \cdot \frac{t d t}{\sqrt{t^{2}-\rho^{2} \sin ^{2} A}}
\end{aligned}
$$




$$
\begin{aligned}
& +\int_{A<\pi / 2} \Omega(\omega) d \omega \int_{\rho+\varepsilon}^{R_{2}(\rho)} \frac{g(t)}{\rho \cos A+\sqrt{t^{2}-\rho^{2} \sin ^{2} A}} \\
& +\int_{A<\pi / 2} \Omega(\omega) d \omega \int_{\rho \sin A}^{\rho-\varepsilon} \frac{t d t}{\frac{\sqrt{t^{2}-\rho^{2} \sin ^{2} A}}{\rho \cos A-\sqrt{t^{2}-\rho^{2} \sin ^{2} A}}} \\
& +\int_{A<\pi / 2} \Omega(\omega) d \omega \int_{\rho \sin A}^{\rho-\varepsilon} \\
& \frac{t d t}{\sqrt{t^{2}-\rho^{2} \sin ^{2} A}} \\
& =I_{1}+I_{2}+I_{3}+I_{4} \cdot \frac{g(t)}{\frac{t d t}{\sqrt{t^{2}-\rho^{2} \sin ^{2} A}}}
\end{aligned}
$$

Here $R_{j}(\rho) \in[R-\rho, R+\rho]$ for $j=1,2$.

The integrand is the same in $I_{1}$ and $I_{2}$, and one finds

$$
\begin{gathered}
I_{1}+I_{2}=\int_{S^{n-1}} \Omega(\omega) d \omega \int_{\rho+\varepsilon}^{R} g(t) \frac{\sqrt{t^{2}-\rho^{2} \sin ^{2} A}-\rho \cos A}{t^{2}-\rho^{2}} \\
\cdot \frac{t d t}{\sqrt{t^{2}-\rho^{2} \sin ^{2} A}}+E .
\end{gathered}
$$

Here the error $E$ is due to the fact that $R_{j}(\rho)$ need not equal $R, j=1,2$. It follows that

$$
|E| \leq \int_{R-\rho \leq|y| \leq R+\rho} \frac{|\Omega(y)|}{|y|^{n}}|f(x-y)| d y \leq C M_{\Omega} f(x),
$$

and Theorem 1 gives the weak type estimate for $\sup _{\varepsilon, R}|E|$. Thus we have

$$
\begin{aligned}
I_{1}+I_{2} & =\int_{\rho+\varepsilon}^{R} g(t) \frac{t}{\rho^{2}-t^{2}} d t \int_{S^{n-1}} \Omega(\omega) \frac{\rho \cos A}{\sqrt{t^{2}-\rho^{2} \sin ^{2} A}} d \omega+E \\
& =J_{1}+E,
\end{aligned}
$$

where we used the equality $\int \Omega(\omega) d \omega=0$. Moreover,

$$
\begin{aligned}
I_{3}+I_{4} & =\int_{0}^{\rho-\varepsilon} g(t) \frac{t}{\rho^{2}-t^{2}} d t \int_{\substack{A<\pi / 2 \\
\sin A<t / \rho}} 2 \Omega(\omega) \frac{\rho \cos A}{\sqrt{t^{2}-\rho^{2} \sin ^{2} A}} d \omega \\
& =J_{2} .
\end{aligned}
$$


That part of $J_{1}$ obtained by integrating only over $t>2 \rho$ is easy to control, since its absolute value is at most

$$
C \int_{2 \rho}^{R}|g(t)| \frac{1}{t} \frac{\rho}{t} d t\|\Omega\|_{1}
$$

It is then enough to observe that

$$
\int_{0}^{\infty} \rho^{n-1} d \rho \int_{2 \rho}^{\infty}|g(t)| \frac{\rho}{t^{2}} d t \leq C \int_{0}^{\infty}|g(t)| t^{n-1} d t .
$$

This takes care of the supremum in $R$.

That part of $J_{2}$ which corresponds to $t<\rho / 2$ can also be easily handled. Indeed, it equals what one gets by restricting the integral defining $\tilde{T}_{\Omega}^{\varepsilon} f(x)$ to the region $|y-x|<\min \{\rho / 2, \rho-\varepsilon\}$. Since $|y| \sim|x|$ in this region, we can dominate by $M_{\Omega} f(x)$ and apply Theorem 1 to get the desired weak type estimate.

The remaining integrals are thus

$$
J_{1}^{\prime}=\int_{\rho+\varepsilon}^{2 \rho} g(t) \frac{t}{\rho^{2}-t^{2}} d t \int_{S^{n-1}} \Omega(\omega) \frac{\rho \cos A}{\sqrt{t^{2}-\rho^{2} \sin ^{2} A}} d \omega
$$

and

$$
J_{2}^{\prime}=\int_{\rho / 2}^{\rho-\varepsilon} g(t) \frac{t}{\rho^{2}-t^{2}} d t \int_{\substack{A<\pi / 2 \\ \sin A<t / \rho}} 2 \Omega(\omega) \frac{\rho \cos A}{\sqrt{t^{2}-\rho^{2} \sin ^{2} A}} d \omega
$$

Notice that the value for $t=\rho$ of the inner integral in $J_{1}^{\prime}$ is

$$
a(\theta)=\int_{S^{n-1}} \Omega(\omega) \operatorname{sgn} \cos A d \omega .
$$

The corresponding quantity for $J_{2}^{\prime}$ is

$$
\int_{A<\pi / 2} 2 \Omega(\omega) d \omega=a(\theta)
$$

because of the vanishing mean value of $\Omega$. Clearly $a$ is a continuous function on $S^{n-1}$. 
If we replace the inner integrals of $J_{1}^{\prime}$ and $J_{2}^{\prime}$ by $a(\theta)$, the resulting expressions will add up to

$$
a(\theta) \int_{[\rho / 2,2 \rho] \backslash[\rho-\varepsilon, \rho+\varepsilon]} g(t) \frac{t}{\rho^{2}-t^{2}} d t .
$$

This integral is a truncation of a smooth principal value singular integral on $\mathbb{R}_{+}$. By standard methods, it can be shown to define a weak type $(1,1)$ operator for the measure $t^{n-1} d t$. So does the corresponding maximal singular integral, defined as the supremum in $\varepsilon$ of the integral.

Since $a$ is a bounded function, we also get a bounded operator from $L^{1}\left(t^{n-1} d t\right)$ into $L^{1, \infty}\left(\mathbb{R}^{n}\right)$.

Thus, to prove Proposition 3, it only remains to estimate the difference operators arising when we subtract $a(\theta)$ from the inner integrals in $J_{1}^{\prime}$ and $J_{2}^{\prime}$. For these operators, we shall actually derive strong type estimates.

For the case of $J_{1}^{\prime}$, we write

$$
\begin{aligned}
\left|\frac{\rho \cos A}{\sqrt{t^{2}-\rho^{2} \sin ^{2} A}}-\operatorname{sgn} \cos A\right| & =\left|\rho \cos A\left(\frac{1}{\sqrt{t^{2}-\rho^{2} \sin ^{2} A}}-\frac{1}{\rho|\cos A|}\right)\right| \\
& \leq \rho|\cos A| \frac{\sqrt{t^{2}-\rho^{2} \sin ^{2} A}-\rho|\cos A|}{\rho|\cos A| \sqrt{t^{2}-\rho^{2} \sin ^{2} A}} \\
& \leq \frac{t^{2}-\rho^{2}}{t^{2}-\rho^{2} \sin ^{2} A},
\end{aligned}
$$

where we multiplied and divided by the conjugate quantity of the numerator, to get the last inequality. Our difference operator is thus controlled by

$$
V_{1} g(\rho, \theta)=\int_{\rho}^{2 \rho}|g(t)| t d t \int_{S^{n-1}}|\Omega(\omega)| \frac{d \omega}{t^{2}-\rho^{2} \sin ^{2} A}
$$

One finds

$$
\begin{aligned}
\int_{S^{n-1}} V_{1} g(\rho, \theta) & d \theta \\
& \leq \int_{\rho}^{2 \rho}|g(t)| t d t \int_{S^{n-1}}|\Omega(\omega)| d \omega \int_{S^{n-1}} \frac{d \theta}{t^{2}-\rho^{2} \sin ^{2} A}
\end{aligned}
$$


Writing $s=t / \rho \in(1,2)$, we see that the innermost integral here is

$$
\begin{aligned}
C \rho^{-2} \int_{0}^{\pi / 2} \frac{\sin ^{n-2} \alpha d \alpha}{s^{2}-\sin ^{2} \alpha} & =C \rho^{-2} \int_{0}^{1} \frac{u^{n-2} d u}{\sqrt{1-u^{2}}\left(s^{2}-u^{2}\right)} \\
& \leq C \rho^{-2} \int_{0}^{1} \frac{d u}{\sqrt{1-u}(s-u)} \\
& =C \rho^{-2} \int_{0}^{1} \frac{d v}{\sqrt{v}(s-1+v)} \\
& =C \rho^{-2}\left(\int_{0}^{s-1}+\int_{s-1}^{1}\right) \\
& \leq \frac{C \rho^{-2}}{\sqrt{s-1}} \\
& =\frac{C \rho^{-3 / 2}}{\sqrt{t-\rho}}
\end{aligned}
$$

This implies

$$
\begin{aligned}
& \int_{0}^{\infty} \rho^{n-1} d \rho \int_{S^{n-1}} V_{1} g(\rho, \theta) d \theta \\
& \leq C \int_{0}^{\infty}|g(t)| t d t \int_{t / 2}^{t} \rho^{n-1-3 / 2} \frac{d \rho}{\sqrt{t-\rho}}\|\Omega\|_{1} \\
& \quad=C \int_{0}^{\infty}|g(t)| t^{n-1} d t\|\Omega\|_{1} .
\end{aligned}
$$

Since $V_{1} g$ does not depend on $\varepsilon$, this is the desired strong type $(1,1)$ estimate.

To deal with the difference operator coming from $J_{2}^{\prime}$, we observe that, almost as in the case of $J_{1}^{\prime}$,

$$
\begin{aligned}
\mid \int_{\substack{A<\pi / 2 \\
\sin A<t / \rho}} 2 \Omega(\omega) & \frac{\rho \cos A}{\sqrt{t^{2}-\rho^{2} \sin ^{2} A}} d \omega-\int_{A<\pi / 2} 2 \Omega(\omega) d \omega \mid \\
& \leq 2 \int_{\substack{A<\pi / 2 \\
\sin A<t / \rho}}|\Omega(\omega)| \frac{\rho^{2}-t^{2}}{\rho \cos A \sqrt{t^{2}-\rho^{2} \sin ^{2} A}} d \omega \\
& +2 \int_{\substack{A<\pi / 2 \\
\sin A>t / \rho}}|\Omega(\omega)| d \omega \\
& =K_{1}+K_{2} .
\end{aligned}
$$


With $s=t / \rho \in(1 / 2,1)$, we now get

$$
\begin{aligned}
& \int_{S^{n-1}} d \theta \int_{\rho / 2}^{\rho-\varepsilon}|g(t)| \frac{t}{\rho^{2}-t^{2}} d t K_{1} \\
& \leq 2 \int_{\rho / 2}^{\rho}|g(t)| t d t \rho^{-2} \int_{S^{n-1}}|\Omega(\omega)| d \omega \int_{\substack{A<\pi / 2 \\
\sin A<s}} \frac{d \theta}{\cos A \sqrt{s^{2}-\sin ^{2} A}} .
\end{aligned}
$$

Here the innermost integral is

$$
\begin{aligned}
C \int_{0}^{\arcsin s} \frac{\sin ^{n-2} \alpha d \alpha}{\cos \alpha \sqrt{s^{2}-\sin ^{2} \alpha}} & =C \int_{0}^{s} \frac{u^{n-2} d u}{\left(1-u^{2}\right) \sqrt{s^{2}-u^{2}}} \\
& \leq C \int_{0}^{s} \frac{d u}{(1-u) \sqrt{s-u}} \\
& =C \int_{0}^{s} \frac{d u}{(1-s+u) \sqrt{u}} \\
& \leq \frac{C}{\sqrt{1-s}} .
\end{aligned}
$$

This implies

$$
\begin{aligned}
\int_{0}^{\infty} \rho^{n-1} d \rho \int_{S^{n-1}} d \theta & \int_{\rho / 2}^{\rho}|g(t)| \frac{t}{\rho^{2}-t^{2}} d t K_{1} \\
& \leq C \int_{0}^{\infty}|g(t)| t d t \int_{t}^{2 t} \rho^{n-1-2+1 / 2} \frac{d \rho}{\sqrt{\rho-t}}\|\Omega\|_{1} \\
& \leq C \int_{0}^{\infty}|g(t)| t^{n-1} d t\|\Omega\|_{1} .
\end{aligned}
$$

Similarly,

$$
\begin{aligned}
\int_{S^{n-1}} d \theta \int_{\rho / 2}^{\rho-\varepsilon} & |g(t)| \frac{t}{\rho^{2}-t^{2}} d t K_{2} \\
& \leq 2 \int_{\rho / 2}^{\rho}|g(t)| \frac{t}{\rho^{2}-t^{2}} d t \int_{S^{n-1}}|\Omega(\omega)| d \omega \int_{\substack{A<\pi / 2 \\
\sin A>s}} d \theta
\end{aligned}
$$

Here the innermost integral is found to be $\mathrm{O}(\sqrt{1-s})$. Integrating the above against $\rho^{n-1} d \rho$, we get at most

$$
C \int|g(t)| t^{n-1} d t\|\Omega\|_{1},
$$


as before. This strong type estimate ends the proof of Proposition 3 .

Proof of Proposition 4. Observe that $T_{\Omega}^{\varepsilon, R} f(x)-\tilde{T}_{\Omega}^{\varepsilon, R} f(x)$ is independent of $R$. One has

$$
D_{\Omega}^{*} f(x) \leq \sup _{\varepsilon>0} \int_{\substack{|y|>\varepsilon \\|| x-y|-| x||<\varepsilon}} \frac{|\Omega(y)|}{|y|^{n}}|f(x-y)| d y .
$$

We assume that $f, g \geq 0$. Notice that $r=\varepsilon$ is equivalent to $t=t_{\varepsilon}$, where

$$
t_{\varepsilon}^{2}=\rho^{2}+\varepsilon^{2}-2 \rho \varepsilon \cos A .
$$

One can assume that $\varepsilon<\rho / 2$, since otherwise the integral in (3.4) is taken over a region where $\varepsilon<|y|<C \varepsilon$. Then the rough maximal operator of Theorem 1 applies.

As in the preceding proof, we write the integral in (3.4) in polar coordinates and replace the integration in $r$ by integration in $t$. Again, we divide the resulting integral into four parts, though not quite in the same way as before. For the supremum of each part, we shall derive a strong or weak type $(1,1)$ estimate.

Part 1: $A>\pi / 2$. Then $\cos A<0$, and $t>\rho$. This part of the integral in (3.4) is dominated in absolute value by

$$
\begin{gathered}
\int_{A>\pi / 2}|\Omega(\omega)| d \omega \int_{t_{\varepsilon}}^{\rho+\varepsilon} \frac{g(t)}{\rho \cos A+\sqrt{t^{2}-\rho^{2} \sin ^{2} A}} \frac{t d t}{\sqrt{t^{2}-\rho^{2} \sin ^{2} A}} \\
\quad=\int_{A>\pi / 2}|\Omega(\omega)| d \omega \int_{t_{\varepsilon}}^{\rho+\varepsilon} \frac{\rho|\cos A|+\sqrt{t^{2}-\rho^{2} \sin ^{2} A}}{t^{2}-\rho^{2}} \\
\quad \frac{g(t) t d t}{\sqrt{t^{2}-\rho^{2} \sin ^{2} A}} \\
\leq 2 \int_{A>\pi / 2}|\Omega(\omega)| d \omega \int_{t_{\varepsilon}}^{\rho+\varepsilon} \frac{g(t) t d t}{t^{2}-\rho^{2}},
\end{gathered}
$$

since here $\rho|\cos A| \leq \sqrt{t^{2}-\rho^{2} \sin ^{2} A}$.

The last inner integral is no larger than

$$
\int_{t_{\varepsilon}}^{\rho+\varepsilon} g(t) \frac{d t}{t-\rho} \leq \int_{\rho}^{\rho+\varepsilon} g(t) \min \left\{\frac{1}{t-\rho}, \frac{1}{t_{\varepsilon}-\rho}\right\} d t .
$$


Since the minimum here is decreasing in $t$ for $\rho<t<\rho+\varepsilon$, it is well known that the right hand integral is dominated by the maximal function of $g$ at $\rho$ times

$$
\int_{\rho}^{\rho+\varepsilon} \min \left\{\frac{1}{t-\rho}, \frac{1}{t_{\varepsilon}-\rho}\right\} d t=1+\log \frac{\varepsilon}{t_{\varepsilon}-\rho}
$$

Instead of the ordinary maximal function $M g(\rho)$, we can here use

$$
M_{\ell} g(\rho)=M\left(g \chi_{[\rho / 2,2 \rho]}\right)(\rho)
$$

since $\varepsilon<\rho / 2$. Because of (3.5), we have

$$
\log \frac{\varepsilon}{t_{\varepsilon}-\rho}=\log \frac{\varepsilon\left(t_{\varepsilon}+\rho\right)}{\varepsilon^{2}+2 \rho \varepsilon|\cos A|} \leq \log \frac{1}{|\cos A|}
$$

Altogether, the expressions in (3.6) are majorized by

$$
2 M_{\ell} g(\rho) \int_{S^{n-1}}|\Omega(\omega)|\left(1+\log \frac{1}{|\cos A|}\right) d \omega
$$

Here the first factor is in $L^{1, \infty}\left(\rho^{n-1} d \rho\right)$ and the second in $L^{1}\left(S^{n-1}\right)$ as a function of $\theta$, as shown via Fubini's theorem. A product of this type belongs to $L^{1, \infty}\left(\rho^{n-1} d \rho d \theta\right)$. Since the product is independent of $\varepsilon$, this ends Part 1.

Part 2: $A<\pi / 2$ and $r<(\rho \cos A) / 2$. Since

$$
r=\rho \cos A-\sqrt{t^{2}-\rho^{2} \sin ^{2} A},
$$

this implies

$$
\sqrt{t^{2}-\rho^{2} \sin ^{2} A}>\frac{1}{2} \rho \cos A .
$$

We can assume that

$$
\frac{1}{2} \rho \cos A>\varepsilon,
$$


because otherwise we get nothing. The part of the integral in (3.4) we get is

$$
\begin{aligned}
& \int_{A<\pi / 2}|\Omega(\omega)| d \omega \int_{\rho-\varepsilon}^{t_{\varepsilon}} \frac{g(t)}{\rho \cos A-\sqrt{t^{2}-\rho^{2} \sin ^{2} A}} \frac{t d t}{\sqrt{t^{2}-\rho^{2} \sin ^{2} A}} \\
& =\int_{A<\pi / 2}|\Omega(\omega)| d \omega \int_{\rho-\varepsilon}^{t_{\varepsilon}} \frac{\rho \cos A+\sqrt{t^{2}-\rho^{2} \sin ^{2} A}}{\rho^{2}-t^{2}} \frac{g(t) t d t}{\sqrt{t^{2}-\rho^{2} \sin ^{2} A}} \\
& \leq C \int_{A<\pi / 2}|\Omega(\omega)| d \omega \int_{\rho-\varepsilon}^{t_{\varepsilon}} \frac{g(t) t d t}{\rho^{2}-t^{2}}
\end{aligned}
$$

the last step because of (3.7).

We proceed as in Part 1. The logarithm to be estimated is now

$$
\log \frac{\varepsilon}{\rho-t_{\varepsilon}}=\log \frac{\varepsilon\left(\rho+t_{\varepsilon}\right)}{2 \rho \varepsilon \cos A-\varepsilon^{2}} .
$$

By means of (3.8), we get rid of the $\varepsilon^{2}$ term in the denominator, and the $\operatorname{logarithm}$ is seen to be dominated by $\log (1 / \cos A)$. The rest is like Part 1.

Part 3: $A<\pi / 2$ and $(\rho \cos A) / 2<r<2 \rho \cos A$. This part of the integral in (3.4) is dominated by the rough maximal function $M_{\Omega}^{*} f(x)$. We apply Theorem 1.

Part 4: $A<\pi / 2$ and $r>2 \rho \cos A$. Notice that this inequality for $r$ is equivalent to $t>\rho$. We can assume that $A>\pi / 4$, because otherwise $\rho / C \leq r \leq C \rho$ for some $C$, and $M_{\Omega}^{*}$ will apply.

The integral we now get is

$$
\begin{gathered}
\int_{\pi / 4<A<\pi / 2}|\Omega(\omega)| d \omega \int_{\rho}^{\rho+\varepsilon} \frac{g(t)}{\rho \cos A+\sqrt{t^{2}-\rho^{2} \sin ^{2} A}} \frac{t d t}{\sqrt{t^{2}-\rho^{2} \sin ^{2} A}} \\
\leq \int_{\pi / 4<A<\pi / 2}|\Omega(\omega)| d \omega \int_{\rho}^{2 \rho} \frac{g(t) t d t}{t^{2}-\rho^{2} \sin ^{2} A} .
\end{gathered}
$$

Notice that the last expression does not contain $\varepsilon$. Its integral with 
respect to $d x=C \rho^{n-1} d \rho d \theta$ is

$$
\begin{gathered}
C \int_{0}^{\infty} \rho^{n-1} d \rho \int_{S^{n-1}} d \theta \int_{\pi / 4<A<\pi / 2}|\Omega(\omega)| d \omega \int_{\rho}^{2 \rho} \frac{g(t) t d t}{t^{2}-\rho^{2} \sin ^{2} A} \\
\leq C \int_{0}^{\infty} g(t) t d t \int_{S^{n-1}}|\Omega(\omega)| d \omega \int_{t / 2}^{t} \rho^{n-1} d \rho \\
\cdot \int_{\pi / 4<A<\pi / 2} \frac{d \theta}{t^{2}-\rho^{2}+\rho^{2} \cos ^{2} A}
\end{gathered}
$$

The innermost integral here is

$$
C \int_{\pi / 4}^{\pi / 2} \frac{\sin ^{n-2} \alpha d \alpha}{t^{2}-\rho^{2}+\rho^{2} \cos ^{2} \alpha} \leq C \int_{0}^{1 / \sqrt{2}} \frac{d u}{t^{2}-\rho^{2}+\rho^{2} u^{2}} \leq \frac{C}{\rho \sqrt{t^{2}-\rho^{2}}} .
$$

It follows that the fourfold integral is no larger than

$$
C \int g(t) t^{n-1} d t\|\Omega\|_{1}
$$

This ends Part 4 and the proof of Proposition 4.

For the $L^{p}$ part of Theorem 2, it is clearly enough to prove versions of Propositions 3 and 4 with strong type $(p, p)$ instead of weak type $(1,1)$. This requires only small modifications of the proofs just given. For instance, in the proof of Proposition 3 one obtains several strong type $(1,1)$ inequalities by integrating various expressions with respect to $\rho^{n-1} d \rho d \theta$. For the $L^{p}$ inequality, one can instead estimate these expressions by quantities like

$$
C M_{\ell} g(\rho) \int_{S^{n-1}}|\Omega(\omega)|\left(1+\log \frac{1}{|\cos A|}\right) d \omega,
$$

which is in $L^{p}\left(\rho^{n-1} d \rho d \theta\right)$ if $g \in L^{p}\left(\rho^{n-1} d \rho d \theta\right)$. We leave the details of the rest of the $L^{p}$ case to the reader.

This ends the proof of Theorem 2 .

\section{References.}

[C-Z] Calderón, A. P., Zygmund, A., On singular integrals. Amer. J. Math. 78 (1956), 289-309. 
[Ch-RF] Christ, M., Rubio de Francia, J. L., Weak type $(1,1)$ bounds for rough operators II. Invent. Math. 93 (1988), 225-237.

[D-RF] Duoandikoetxea, J., Rubio de Francia, J. L., Maximal and singular integral operators via Fourier transforms estimates. Invent. Math. $\mathbf{8 4}$ (1986), 541-561.

[H] Hofmann, S., Weak $(1,1)$ boundedness of singular integrals with nonsmooth kernel. Proc. Amer. Math. Soc. 103 (1988), 260-264.

[Se] Seeger, A., Singular integral operators with rough convolution kernels. J. Amer. Math. Soc. 9 (1996), 95-105.

[S-S] Sjögren, P., Soria, F., Weak type $(1,1)$ estimates for some integral operators related to rough maximal functions. Israel J. Math. 95 (1996), 211-229.

[St] Stein, E. M., Singular Integrals and differentiability properties of functions. Princeton University Press, 1970.

Recibido: 21 de junio de 1.995

Revisado: 6 de noviembre de 1.995

Peter Sjögren

Department of Mathematics Chalmers University of Technology and Göteborg University

S-412 96 Göteborg, SWEDEN peters@math. chalmers.se

and

Fernando Soria

Departamento de Matemáticas Universidad Autónoma de Madrid

E-28049 Madrid, ESPAÑ

fernando.soria@uam.es

Both authors were partially supported by the Spanish D.G.I.C.Y.T., the first one during a sabbatical leave. They were also supported under the E.U. contract ERBCHRXCT 930083. 